\title{
CASowary: CRISPR-Cas13 guide RNA predictor for transcript depletion
}

2 Alexander Krohannon ${ }^{1}$, Mansi Srivastava ${ }^{1}$, Simone Rauch ${ }^{2}$, Rajneesh Srivastava ${ }^{1}$, Bryan C. Dickinson²,

3 Sarath Chandra Janga ${ }^{1,3,4^{*}}$

4

$5 \quad{ }^{1}$ Department of BioHealth Informatics, School of Informatics and Computing, Indiana University

6 Purdue University, 719 Indiana Ave St 319, Walker Plaza Building, Indianapolis, Indiana 46202

$7 \quad{ }^{2}$ Department of Chemistry, The University of Chicago, Chicago, Illinois, USA

$8 \quad{ }^{3}$ Center for Computational Biology and Bioinformatics, Indiana University School of Medicine, 5021

9 Health Information and Translation Sciences (HITS), 410 West $10^{\text {th }}$ Street, Indianapolis, Indiana 46202

$10{ }^{4}$ Department of Medical and Molecular Genetics, Indiana University School of Medicine, Medical

11 Research and Library Building, 975 West Walnut Street, Indianapolis, Indiana, 46202

12

13 *Correspondence should be addressed to:

14

15 Sarath Chandra Janga (scjanga@iupui.edu)

16719 Indiana Avenue Ste 319,

17 Walker Plaza Building

18 Indianapolis, Indiana -46202

19 Tel: +1-317-278-4147, Fax: +1-317-278-9201 


\section{Abstract}

Recent discovery of the gene editing system - CRISPR (Clustered Regularly Interspersed Short Palindromic Repeats) associated proteins (Cas), has resulted in its widespread use for improved understanding of a variety of biological systems. Cas13, a lesser studied Cas protein, has been repurposed to allow for efficient and precise editing of RNA molecules. The Cas13 system utilizes base complementarity between a crRNA/sgRNA (crispr RNA or single guide RNA) and a target RNA transcript, to preferentially bind to only the target transcript. Unlike targeting the upstream regulatory regions of protein coding genes on the genome, the transcriptome is significantly more redundant, leading to many transcripts having wide stretches of identical nucleotide sequences. Transcripts also exhibit complex three-dimensional structures and interact with an array of RBPs (RNA Binding Proteins), both of which further limit the scope of effective target sequences. As a result, there currently exists no method to predict whether a specific sgRNA will effectively knockdown a transcript. Here we present a novel machine learning and computational tool, CASowary, to predict the efficacy of a sgRNA. We used publicly available RNA knockdown data from Cas13 characterization experiments for 555 sgRNAs targeting the transcriptome in HEK293 cells, in conjunction with transcriptome-wide protein occupancy information on RNA. Our model utilizes a Decision Tree architecture with a set of 112 sequence and target availability features, to classify sgRNA efficacy into one of four classes, based upon expected level of target transcript knockdown. After accounting for noise in the training data set, the noise-normalized accuracy exceeds 70\%. Additionally, highly effective sgRNA predictions have been experimentally validated using an independent RNA targeting Cas system - CIRTS, confirming the robustness and reproducibility of our model's sgRNA predictions. Utilizing transcriptome wide protein occupancy map generated using POP-seq in Hela cells against publicly available protein-RNA interaction map in Hek293 cells, we show that CASowary can predict high quality guides for numerous transcripts in a cell line specific manner. Application of CASowary to whole transcriptomes should enable rapid deployment of CRISPR/Cas13 systems, facilitating the development of therapeutic interventions linked with aberrations in RNA regulatory processes.

Keywords: CRISPR/Cas13, mRNA regulation, gene editing, functional genomics, machine learning, protein expression 
49

\section{Background}

Gene editing technologies have played an increasingly important role in numerous life science domains in the recent years, especially in the fields of biology, biotechnology, and medicine (1). At the center of many of these discoveries is the CRISPR/Cas9 gene editing system (2). This system has allowed an unprecedented level of accurate and precise editing of the genome. Several limitations have been recognized with the use of CRISPR/Cas9 system: the requirement of a PAM (protospacer adjacent motif) sequence adjacent to the target gene sequence, reliance on dynamic DNA repair procedures (3), and its inability to facilitate tissue specific alterations (4). However, other Cas proteins are being identified and repurposed as systems for genome and transcriptome editing (5).

One such class of protein, Cas13, has been modified to directly edit RNA transcripts (5). Much like Cas9, the Cas13 system is a two-component system: the Cas13 enzyme and sgRNA. After binding to the sgRNA, the Cas13 complex probes the cellular RNA molecules for a sequence complementary to the 28-nucleotide spacer sequence of the bound sgRNA. Once identified, the enzyme binds to the RNA molecule for its catalytic cleavage, rendering it ineffective and facilitating RNA degradation. Some of the most promising aspects of this system are the independence from the PAM motif restriction and the potential for designing guide sequences for enabling tissue specific transcript knockdowns.

While the Cas13 system does offer some distinct advantages over the Cas9 system, it also poses some unique challenges. First and foremost, most of the transcriptome remains unknown, owing to poor understanding of various post transcriptional processes. RNA molecules can also adopt a variety of complex three-dimensional structures through networks of intramolecular interactions. Additionally, there are a variety of proteins, RNA binding proteins (RBPs), which bind to various regions of RNA molecules. Together the irregular complex structure of RNAs and RBP binding act to limit the number 
71 of stretches available for complementary base pairing. Therefore, a tool to predict the efficacy of a 72 given sgRNA is desirable.

73

To that end CASowary was developed as a first of its kind sgRNA efficacy predictor.

Although several previous studies have focused on creating software to predict sgRNA for CRISPR

Cas9, to our knowledge, there have been no previous attempts for doing such for CRISPR Cas13 (6-8).

CASowary was written in python3 and uses a variety of functions from various libraries: vector

operations form numpy, statistical analysis from scipy, machine learning utilities from sklearn, and data visualization from seaborn and matplotlib (9-13). The development and validation of CASowary took place over three distinct phases: Data Collection and Integration, Feature Selection, and Model Generation and Benchmarking (Fig 1). Three different types of data were utilized by the model for predictions - targeted RNA knockdown experiments (14), transcriptome-wide protein occupancy information (15), and sgRNA spacer sequence alignment data. Feature selection took place through a variety of steps including composition analysis, k-mer capture, and evaluating feature significance and contribution. The model was validated using both 3-fold and 5-fold cross-validation. Additionally, the model's predictions were verified through an experimental protocol with an orthogonal CRISPR based system (16). The model was then applied to all transcripts from among 5900 random genes, to determine any biological relevance of the model's predictions.

\section{Implementation}

\section{Genome-scale sequence data for CRISPR-Cas13}

Utilizing the Cas13 human transcript knockdown experiments from Abudayyeh et al (14), we sought to develop a machine learning model that predicts the effectiveness of a given sgRNA at knocking down a target transcript. Firstly, we investigated the sequence composition i.e. mono-, di-, 
94 and tri-nucleotide compositions for all 555 guide-RNAs (or sgRNA) at each position along the 28-nt

95 length. We obtained a list of over and under-represented k-mers (chi-squared test) at each location

96 across the spacer sequence of the sgRNA (Fig 2 A-B). Afterwards, sgRNAs were then partitioned into

97 distinct groups based upon their nucleotide composition at a specific location; in order to perform a

98 Kruskal Wallis (17) test. Sets of positions with Kruskal values less than 0.05 were correlated with

99

100

101

102

103

104

105

106

107

108

109

110

111

112

113

114

115

116

nucleotides that were over or under-represented at a particular location.

The significance of each k-mer composition feature was then evaluated using the univariate linear regression module from sklearn. Next, all sequence features with p-values greater than 0.05 were removed (Fig 2C). Wary of being too inclusive with all statistically significant features, two additional subsets of these features were also considered, using a Z-score analysis on the negative log of p-values. Using 2 and 3 as the cutoff values, two sets of highly correlated statistical features were generated. In addition to this, a Gini score analysis was performed on each k-mer using the Decision Tree (18) and Random Forest (19) machine learning modules from sklearn. A similar approach was utilized by Fusi et al (20) for determining the most important features for CRISPR/Cas9 efficiency.

\section{Protein-RNA occupancy profile}

In addition to the sequence composition features, we included transcriptome-wide occupancy as a feature into the model. To do so, we downloaded the transcriptome-wide protein occupancy data (raw reads in the FASTQ format) of HEK293 cells from Schueler et al (15). Reads were checked for adapter content and overall quality using Fastqc (21), trimmed using Trim Galore $(22,23)$, and aligned to the human reference transcriptome (a combination of hg38 cDNA and ncRNA downloaded from Biomart (24) using hisat2 (25). After sequence alignment, peak calling was performed using macs2 (26), the resulting .xls file was used as an input for the model. Each guide sequence was also aligned to the 
human reference transcriptome, using tophat (27), allowing for 2 mismatches, the maximum number of mismatches tolerated by the CRISPR cas13 system (14). The indexed position of each guide on the target transcript was compared with the protein occupancy information for any overlap. The amount of overlap was recorded as a percentage of length of the guide and incorporated as a feature in the model.

\section{Composition features}

In addition to the k-mer composition and occupancy features, a variety of other features were also included. These include guide spacer percent composition for each nucleic acid, guide location along the length of the transcript, and the number of complementary sequences in the reference transcriptome. Previous studies $(28,29)$ have shown that RNA base composition plays a crucial role in not just the long term stability of the polynucleotide, but also in the activity of the Cas13 system. It is widely believed that the ends of transcripts, both $5^{`}$ and $3 `$ are highly structured, both to protect the transcript from degradation and to facilitate movement to different cellular compartments. This information was incorporated as a feature into the model, by calculating the midpoint of the complementary region of the transcript, and normalizing by the length of the target transcript. Despite the length of the guide, it is possible for guides to be complementary to different regions of different transcripts. This redundancy in targets, would possibly lead to off target effects, and significantly reduce the system's ability to deplete a target transcript. To capture this in the model, the number of different hits returned from the tophat alignment for each guide was also recorded as a feature.

\section{Model architecture and feature selection}

The occupancy and composition features, were then combined with the several sets of k-mer features (significant, Z-score $>2$, Z-score $>3$, decision tree Gini, and random forest Gini) and tested using a variety of machine learning algorithms, to determine the optimal combination. Each framework 
141

142

143

144

145

146

147

148

149

150

151

152

153

154

155

156

157

158

159

160

161

was evaluated based on their ability to accurately classify guides into one of four classes $(0-3)$, based upon the quartile of transcript expression. This was tested by utilizing two different methods of crossvalidation: 3-fold and 5-fold. For the 3-fold cross-validation, the experimental replicates were divided into separate folds, with two replicates serving as the testing data, and the other serving as an independent data set. For the 5-fold cross-validation the data from all 3 replicates were randomized, with $80 \%$ selected as training data and $20 \%$ selected for testing. The average values of the three different 3-fold experiments are presented in Table 1, as well as the average value of 100 different 5fold experiments.

Due to the experimental noise native to the data source methodology, a significant amount of the experimental replicates for a specific guide differed significantly in transcript expression, often by more than $25 \%$. This discordance in the training data lead to the model receiving different labels for the same set of training features, imposing a hard cap to the model's cross-validation performance. To account for this, the models were evaluated based upon noise-normalized accuracy. The noisenormalized maximum was calculated by counting the number of occurrences where one experimental replicate differed in transcript expression quartile, with another replicate of the same experiment. Put more formally by, computing the size of the set of tuples $(\mathrm{i}, \mathrm{j})$ such that $\mathrm{x}_{\mathrm{i}}=\mathrm{x}_{\mathrm{j}}$ and $\mathrm{y}_{\mathrm{i}} \neq \mathrm{y}_{\mathrm{j}}$, divided by the size of the set (i,j), and subtracting that value from 1 (where $\mathrm{x}$ and $\mathrm{y}$ correspond to the model input data and the label, respectively). The total model accuracy was then divided by the noise-normalized maximum to create the noise-normalized accuracy value.

$$
\begin{gathered}
1.1-\frac{\left\|(i, j) \mid x_{i}=x_{j} \wedge y_{i} \neq y_{j}\right\|}{\|(i, j)\|}=\max _{n n} \\
2 . \frac{a c c}{\max _{n n}}=a c c_{n n}
\end{gathered}
$$



another 100 times. The difference in model performance between the mean model accuracy and the background was taken to be the result of the removed feature (Fig 2D). small RNA library prep kit (Illumina). All libraries were barcoded and sequenced in parallel on a Nextseq platform for 400 million reads to obtain 75 bp single end reads.

\section{Results}

CASowary takes a list of gene names as input and exports a list of sgRNA sequences predicted 
185

186

187

188

189

190

191

192

193

194

195

196

197

198

199

200

201

202

203

204

205

206

207

208

the reference transcriptome using tophat, allowing for 2 mismatches, to create a BAM file. The resulting BAM file is converted to a BED file using bedtools (31). That BED file is then fed into the model where it classifies each guide; and outputs a separate text file for each transcript mapping to an input gene name, containing all highly effective guide sequences ranked upon model confidence in its classification.

Our tool uses a Decision Tree architecture and set of features based upon Random Forest Gini analysis to classify a sgRNA into 1 of 4 classes, based upon predicted transcript knockdown efficiency. Each class represents a specific quartile of normalized expression $(0: 0-0.25,1: 0.25-0.5,2: 0.5-$ 0.75, and 3: $0.75-1)$. Guides belonging to class 0 and 1 were categorized as highly efficient and efficient, while classes 2 and 3 correspond to inefficient and highly inefficient, respectively. Utilizing 5-fold and 3-fold cross-validation, this model was benchmarked with noise-normalized accuracy of $70.1 \%$ and $74.3 \%$ respectively $(69.2 \%$ and $71.5 \%$ without accounting for noise in the source data). A small amount of overfitting was observed in the 3-fold cross-validation, due to the identical model inputs, so $70.1 \%$ was believed to be the most accurate measure of the model's performance.

Using one class vs all pairwise comparisons, a Receiver Operating Characteristic (ROC) curve for the model was created (Fig 3). Calculating the Area Under the Curve (AUC) for each class revealed that the model performed best predictions for highly efficient and highly inefficient guides (0: 0.949, 1: 0.869, 2: 0.753 , and 3: 0.839). These numbers clearly illustrate an increased sensitivity in model's predictions for highly efficient and efficient class of guides, maximizing its effectiveness.

We performed a predictive analysis against 5 different genes, including the SMARCA4 gene.

We conducted a series of characterization experiments using CIRTS (CRISPR-Cas-inspired RNA targeting system), an orthogonal RNA-targeting system $(16,32)$. A series of guides targeting a single SMARCA4 transcript (ENST00000344626.9) were obtained from IDT and the transcript depletion experiment data was generated and analyzed for a select set of sgRNA predictions (Fig 4) (16). 
bioRxiv preprint doi: https://doi.org/10.1101/2021.07.26.453663; this version posted July 27, 2021. The copyright holder for this preprint (which

was not certified by peer review) is the author/funder, who has granted bioRxiv a license to display the preprint in perpetuity. It is made available under aCC-BY-NC-ND 4.0 International license.

209 Comparing our model's predictions of high (best) and low (worst) efficiency guides and the

210 experimental results of CIRTS showed a very high correlation (Fig 4). This experimental confirmation

211 illustrates the robust and reproducible nature of predictions made by CASowary using an orthogonal

212 RNA editing system to Cas13.

213

During the development of CASowary, we observed that specific classes of guides exhibited

preferential patterns across the length of the transcripts. To confirm this trend, a comprehensive

215 analysis of the predictions for a random assortment of 5900 gene transcripts were performed, resulting

in 12.7 million mapped guides. All guides of a specific class were then grouped and plotted against

217 their corresponding location in the transcript, from 5 ` to $33^{`}$ direction, by normalizing the length to

understand locational preferences for various classes of guides. This analysis revealed that the majority

of the guides were predicted to be inefficient, either categorized as Highly Inefficient or Inefficient

(90.7\%) (Fig 5 C-D). In addition, our data suggests that efficient guides (Efficient and Highly Efficient)

primarily reside in the intermediate regions of the transcript, especially between $30 \%$ and $70 \%$ the

length of the transcript (Fig 5A-B). Distribution of the guide locations was similar when we plotted the data for the complete training data (Fig 5A) as well as the computational guide predictions for 5900 genes (Fig 5B). This observation supports the theory that the ends of active mRNAs are highly structured, with multiple binding proteins and other modifications that further limit the binding efficiency of the CRISPR-Cas13 system.

The secondary location for efficient guides, lying between $0 \%$ and $20 \%$ of the transcript (near the 5 ` end) among the computational predictions (Fig 5B), was unexpected and required additional 229 investigation. Of the 5900 genes included in the analysis, transcripts from 4361 different genes 230 included efficient guides in the upper quintet. That subset included 1417 different genes associated with 231 lncRNA (32\%), over 90\% of all genes (1570) associated with lncRNA. The average length of these 232 transcripts (1670 nucleotides) was significantly longer than the average length of all transcripts (1537 
nucleotides) with p-values from Mann-Whitney (33) of 1.34 x $10^{-43}$. This illustrates that longer transcripts are more likely to have guides in this region, and that this region is the prime target for lncRNA depletion.

Next, we investigated the ability of CASowary to generate cell type specific guide predictions by employing the tool to predict guide sequences on the HeLa cell line. To this end, we utilized inhouse phase separation based protein occupancy data for the HeLa cell line, through a method called Protein-Occupancy Profile Sequencing (POP-seq) to map protein-RNA interactions on a transcriptome wide scale (30). Protein RNA-interactions are known to vary from cell type to cell type, which would alter the accessibility feature of the model $(34,35)$.

The reads from the experiment, corresponding to stretches of RNA molecules interacting with proteins, were run through the same computational pipeline as described in methods. The resulting file was substituted for the HEK293 peak file in CASowary's input. A list of 100 candidate genes with differential binding profiles between the HEK293 and the HeLa files was generated by running them through DiffHunter (36). This list of candidate genes was then analyzed using CASowary with the HeLa occupancy profile. Transcript levels were verified by comparing prevalence of reads supporting a specific transcript from RNA-Seq experiments on the wild type of that cell line. This data was obtained for both HEK293 and HeLa cells from the Gene Expression Omnibus (GEO) (37), series accession number GSE146946.

The results of CASowary predictions for the two cell lines were visualized using Integrative Genomics Viewer (38) along with their relative abundance (SRR11304482 and SRR11304484, for HEK293 and HeLa respectively) (39). Comparing the two cell line results revealed high correlation for guides outside of protein occupied regions. However, regions with differential protein binding exhibited a significantly altered pattern of predicted guides across the transcript (Fig 6). The results from the analysis indicate that CASowary's guide predictions are highly sensitive to protein occupancy, 
257 allowing for cell type specific guide predictions. This exciting development illustrates the importance

258 and need for more in-depth protein occupancy protocols to enable guide predictions on gene regulatory

259 regions tailored for specific tissues and cell types.

261 Conclusions

Gene and transcript editing technologies, such as CRISPR and its variant systems, will continue

to evolve for their application, and so too will the demand for computational and predictive tools to

264 improve the efficacy of these methods. We present CASowary as the first of its kind, tool that provides

features, our tool can generate a list of potential sgRNAs predicted to be highly efficient, from among gene therapies and personalized medicine.

Despite the success of the current iteration of our tool, there still remains room for the structure of the target RNA in vivo. There is also a desire to expand the cell and tissue specific predictions, but that requires substantially more protein occupancy information.

\section{Availability and requirements}

CASowary is written in Python, requiring 3.6.8 or above, with some dependencies on Python

\section{Abbreviations:}


280 AUC: Area Under the Curve

281 Cas: CRISPR associated

282 cDNA: complementary DNA

283 CIRTS: CRISPR-Cas-Inspired RNA Targeting System

284 CRISPR: Clustered Regularly Interspersed Short Palindromic Repeats

285 crRNA crispr RNA

286 DNA: DeoxyRibonucleic Acid

287 GEO: Gene Expression Omnibus

288 IDT: Integrated DNA Technologies

289 IGV: Integrative Genomics Viewer

290 lncRNA: long non-coding RNA

291 ncRNA: non-coding RNA

292 NIH: National Institute of Health

293 PAM: Protospacer Adjacent Motif

294 POP-seq: Protein Occupancy Profile sequencing

295 RBP: RNA Binding Protien

296 ROC: Receiver Operator Curve

297 sgRNA: single guide RNA

298

299 Declarations:

300 Ethics approval and consent to participate:

$301 \quad$ NA

302 
303 Consent to publish:

304 NA

305

306 Availability of data and materials:

307 All Pop-seq data generated in this study is deposited under GEO accession number GSE166189.

308 Reviewers can confidentially access the data via https:/www.ncbi.nlm.nih.gov/geo/query/acc.cgi?

$309 \underline{\text { acc }=\text { GSE166189 }}$ and entering token 'mtybqumuzxuzrkz' until the data is made open access.

310

311 Competing interests:

312 The authors report no financial or other conflict of interest relevant to the subject of this article.

314 Funding:

315 This work was supported by the National Institute of General Medical Sciences of the National

316 Institutes of Health under Award Number R01GM123314 and Eli Lilly Research Award Program grant

317 (SCJ). Additional support was provided by National Institute of General Medical Sciences (R35

318 GM119840) and the National Institute of Mental Health (R01 MH122142) of the National Institutes of

319 Health (NIH) (BCD).

321 Author's Contributions:

322 AK wrote the code base, performed the computational benchmarking, and wrote the manuscript. MS

323 performed the laboratory work for POP-seq data generation. SR performed CIRTS experiments to 324 validate guide RNA efficacy. RS analyzed POP-seq data. BCD supervised the CIRTS characterization

325 experiments. SCJ oversaw the creation of the tool, collaboration with external colleagues, and

326 contributed to drafting the manuscript. All authors have read and approved the final manuscript. 
328 Acknowledgments:

329 The authors would like to thank the respective labs of SCJ and BCD for their advice and support over

330 the course of the project. AK, MS, and SCJ would like to thank Dr. Mark Kaplan for providing space

331

332

333

and access to equipment for wet lab experiments.

\section{References}

1. Hsu PD, Lander ES, Zhang F. Development and applications of CRISPR-Cas9 for genome engineering. Cell. 2014 Jun 5;157(6):1262-78.

2. Li J, Shou J, Guo Y, Tang Y, Wu Y, Jia Z, et al. Efficient inversions and duplications of mammalian regulatory DNA elements and gene clusters by CRISPR/Cas9. J Mol Cell Biol. 2015

Aug;7(4):284-98.

3. Yen S-T, Zhang M, Deng JM, Usman SJ, Smith CN, Parker-Thornburg J, et al. Somatic mosaicism and allele complexity induced by CRISPR/Cas9 RNA injections in mouse zygotes. Dev Biol. 2014 Sep 1;393(1):3-9.

4. Burstein D, Harrington LB, Strutt SC, Probst AJ, Anantharaman K, Thomas BC, et al. New CRISPR-Cas systems from uncultivated microbes. Nature. 2017 Feb 9;542(7640):237-41.

5. Cox DBT, Gootenberg JS, Abudayyeh OO, Franklin B, Kellner MJ, Joung J, et al. RNA Editing with CRISPR-Cas13. Science. 2017 Nov 24;358(6366):1019-27.

6. Abadi S, Yan WX, Amar D, Mayrose I. A machine learning approach for predicting CRISPR-Cas9 cleavage efficiencies and patterns underlying its mechanism of action. PLoS Comput Biol [Internet]. 2017 Oct 16 [cited 2021 May 12];13(10). Available from: https://www.ncbi.nlm.nih.gov/pmc/articles/PMC5658169/

7. Chuai G, Ma H, Yan J, Chen M, Hong N, Xue D, et al. DeepCRISPR: optimized CRISPR guide RNA design by deep learning. Genome Biol [Internet]. 2018 Jun 26 [cited 2021 May 12];19. Available from: https:/www.ncbi.nlm.nih.gov/pmc/articles/PMC6020378/

8. Liu Q, Cheng X, Liu G, Li B, Liu X. Deep learning improves the ability of sgRNA off-target propensity prediction. BMC Bioinformatics [Internet]. 2020 Feb 10 [cited 2021 May 12];21. Available from: https:/www.ncbi.nlm.nih.gov/pmc/articles/PMC7011380/

9. Harris CR, Millman KJ, van der Walt SJ, Gommers R, Virtanen P, Cournapeau D, et al. Array programming with NumPy. Nature. 2020;585(7825):357-62. 
10. Virtanen P, Gommers R, Oliphant TE, Haberland M, Reddy T, Cournapeau D, et al. SciPy 1.0: fundamental algorithms for scientific computing in Python. Nat Methods. 2020;17(3):261-72.

11. Pedregosa F, Varoquaux G, Gramfort A, Michel V, Thirion B, Grisel O, et al. Scikit-learn: Machine Learning in Python. J Mach Learn Res. 2011 Nov 1;12(null):2825-30.

12. Waskom ML. seaborn: statistical data visualization. J Open Source Softw. 2021 Apr 6;6(60):3021.

13. Hunter JD. Matplotlib: A 2D Graphics Environment. Comput Sci Eng. 2007 May;9(3):90-5.

14. Abudayyeh OO, Gootenberg JS, Essletzbichler P, Han S, Joung J, Belanto JJ, et al. RNA targeting with CRISPR-Cas13a. Nature. 2017 Oct 12;550(7675):280-4.

15. Schueler M, Munschauer M, Gregersen LH, Finzel A, Loewer A, Chen W, et al. Differential protein occupancy profiling of the mRNA transcriptome. Genome Biol. 2014;15(1):R15.

16. Rauch S, He E, Srienc M, Zhou H, Zhang Z, Dickinson BC. Programmable RNA-guided RNA effector proteins built from human parts. Cell. 2019 Jun 27;178(1):122-134.e12.

17. Kruskal WH, Wallis WA. Use of Ranks in One-Criterion Variance Analysis. J Am Stat Assoc. 1952 Dec 1;47(260):583-621.

18. Krzywinski M, Altman N. Classification and regression trees. Nat Methods. 2017 Aug 1;14(8):757-8.

19. Breiman L. Random Forests. Mach Learn. 2001 Oct 1;45(1):5-32.

20. Fusi N, Smith I, Doench J, Listgarten J. In Silico Predictive Modeling of CRISPR/Cas9 guide efficiency. bioRxiv. 2015 Jun 26;021568.

21. Wingett SW, Andrews S. FastQ Screen: A tool for multi-genome mapping and quality control. F1000Research [Internet]. 2018 Sep 17 [cited 2021 May 12];7. Available from: https://www.ncbi.nlm.nih.gov/pmc/articles/PMC6124377/

22. Martin M. Cutadapt removes adapter sequences from high-throughput sequencing reads. EMBnet.journal. 2011 May 2;17(1):10-2.

23. Krueger F. FelixKrueger/TrimGalore [Internet]. 2021 [cited 2021 May 12]. Available from: https://github.com/FelixKrueger/TrimGalore

24. Kinsella RJ, Kähäri A, Haider S, Zamora J, Proctor G, Spudich G, et al. Ensembl BioMarts: a hub for data retrieval across taxonomic space. Database J Biol Databases Curation [Internet]. 2011 Jul 23 [cited 2021 May 12];2011. Available from: https:/www.ncbi.nlm.nih.gov/pmc/articles/PMC3170168/

25. Kim D, Langmead B, Salzberg SL. HISAT: a fast spliced aligner with low memory requirements. Nat Methods. 2015 Apr;12(4):357-60. 
bioRxiv preprint doi: https://doi.org/10.1101/2021.07.26.453663; this version posted July 27, 2021. The copyright holder for this preprint (which

was not certified by peer review) is the author/funder, who has granted bioRxiv a license to display the preprint in perpetuity. It is made available under aCC-BY-NC-ND 4.0 International license.

26. Zhang Y, Liu T, Meyer CA, Eeckhoute J, Johnson DS, Bernstein BE, et al. Model-based Analysis of ChIP-Seq (MACS). Genome Biol. 2008;9(9):R137.

27. Trapnell C, Pachter L, Salzberg SL. TopHat: discovering splice junctions with RNA-Seq. Bioinformatics. 2009 May 1;25(9):1105-11.

28. Lesnik EA, Freier SM. Relative Thermodynamic Stability of DNA, RNA, and DNA:RNA Hybrid Duplexes: Relationship with Base Composition and Structure. Biochemistry. 1995 Aug 29;34(34):10807-15.

29. Wessels H-H, Méndez-Mancilla A, Guo X, Legut M, Daniloski Z, Sanjana NE. Massively parallel Cas13 screens reveal principles for guide RNA design. Nat Biotechnol. 2020 Jun;38(6):722-7.

30. Srivastava M, Srivastava R, Janga SC. Transcriptome-wide high-throughput mapping of proteinRNA occupancy profiles using POP-seq. Sci Rep [Internet]. 2021 Jan 13 [cited 2021 May 12];11. Available from: https:/www.ncbi.nlm.nih.gov/pmc/articles/PMC7806670/

31. Quinlan AR, Hall IM. BEDTools: a flexible suite of utilities for comparing genomic features. Bioinformatics. 2010 Mar 15;26(6):841-2.

32. Rauch S, Jones KA, Dickinson BC. Small Molecule-Inducible RNA-Targeting Systems for Temporal Control of RNA Regulation. ACS Cent Sci. 2020 Nov 25;6(11):1987-96.

33. Mann HB, Whitney DR. On a Test of Whether one of Two Random Variables is Stochastically Larger than the Other. Ann Math Stat. 1947 Mar;18(1):50-60.

34. Masuda K, Marasa B, Martindale JL, Halushka MK, Gorospe M. Tissue- and age-dependent expression of RNA-binding proteins that influence mRNA turnover and translation. Aging. 2009 Jul 26;1(8):681-98.

35. Mironov A, Denisov S, Gress A, Kalinina OV, Pervouchine DD. An extended catalogue of tandem alternative splice sites in human tissue transcriptomes. PLoS Comput Biol [Internet]. 2021 Apr $7 \quad$ [cited 2021 May 12];17(4). Available from:

https://www.ncbi.nlm.nih.gov/pmc/articles/PMC8055015/

36. Sasanh. Sasanh/diffHunter [Internet]. 2017 [cited 2021 May 12]. Available from: https://github.com/Sasanh/diffHunter

37. Edgar R, Domrachev M, Lash AE. Gene Expression Omnibus: NCBI gene expression and hybridization array data repository. Nucleic Acids Res. 2002 Jan 1;30(1):207-10.

38. Thorvaldsdóttir H, Robinson JT, Mesirov JP. Integrative Genomics Viewer (IGV): highperformance genomics data visualization and exploration. Brief Bioinform. 2013 Mar;14(2):17892.

39. Song Y, Li L, Yang W, Fu Q, Chen W, Fang Z, et al. Sense-antisense miRNA pairs constitute an elaborate reciprocal regulatory circuit. Genome Res. 2020 May;30(5):661-72. 


\section{Figure Legends:} associated with 5900 genes. The results were collected and analyzed for any potential biological

345 relevance for predictions.

Figure 2. K-mer Analysis to study the guide composition. A: Bar plot of the population of sgRNAs that contain a specific dinucleotide at position 8. B: Box plot of target transcript expression values as a significance p-value for all monomers at all positions across the guide. D: Bar plot of the feature contribution score for each feature in the Random Forest gini feature list. 
guide predictions, transcript expression value between 0.25 - 0 (red) and low efficiency CASowary guide predictions, transcript expression value between 0.75 and 1 (blue).

\section{Figure 5. Comparison of Training Data with Gene Predictions: A: Density plot of Efficient (Highly} Efficient and Efficient) and Inefficient (Inefficient and Highly Inefficient) guides from the training data. B: Density plot of Efficient and Inefficient guides from the 5900 random genes. C: Pie chart for the breakdown of guide predictions from the training data. D: Pie chart for the breakdown of the guide predictions from the 5900 random genes.

Figure 6. Cell Line Specific Predictions: IGV tracks for ENST00000554738.5, a protein coding transcript for NRXN3. The top collection of tracks corresponds to protein occupancy (blue), high quality guide locations (transcript expression value between 0 and 0.5) (green), and transcript abundance for HEK293 cell line (gray). The bottom collection of tracks is the same for the HeLa cell line.

\section{Tables}

Table 1. Model Architecture Performance by Feature Set. Distribution of model accuracy using a variety of different architectures and different feature lists for both 5-fold and 3-fold cross validation methods. For KNN and Random Forest, average values for parameters with the highest accuracy are recorded in brackets.

\begin{tabular}{|c|c|c|c|c|c|}
\hline 3-Fold & $\mathrm{P}<0.05$ & $\mathrm{Z}>2$ & $\mathrm{Z}>3$ & Gini & Decision Tree \\
\hline Random Forest & $0.654[41.3]$ & $0.651[46.75]$ & $0.641[45.75]$ & $0.652[43.95]$ & $0.654[41.3]$ \\
\hline KNN & $0.558[3.39]$ & $0.496[9.71]$ & $0.515[8.44]$ & $0.535[3.67]$ & $0.558[3.39]$ \\
\hline SVC [linear] & 0.615 & 0.553 & 0.504 & 0.589 & 0.634 \\
\hline
\end{tabular}


bioRxiv preprint doi: https://doi.org/10.1101/2021.07.26.453663; this version posted July 27, 2021. The copyright holder for this preprint (which was not certified by peer review) is the author/funder, who has granted bioRxiv a license to display the preprint in perpetuity. It is made available under aCC-BY-NC-ND 4.0 International license.

\begin{tabular}{|c|c|c|c|c|c|}
\hline SVC [poly] & 0.593 & 0.535 & 0.501 & 0.562 & 0.574 \\
\hline SVC [sigmoid] & 0.551 & 0.489 & 0.458 & 0.517 & 0.521 \\
\hline SVC [rbf] & 0.563 & 0.504 & 0.469 & 0.537 & 0.541 \\
\hline Decision Tree & 0.634 & 0.636 & 0.634 & 0.640 & 0.637 \\
\hline 5-Fold & $\mathbf{P}<0.05$ & $\mathrm{Z}>\mathbf{2}$ & $\mathrm{Z}>\mathbf{3}$ & Gini & Decision Tree \\
\hline Random Forest & $0.717[55.4]$ & $0.713[57.55]$ & 0.714 [51.1] & $0.716[54.35]$ & $0.717[50.55]$ \\
\hline KNN & $0.715[2]$ & $0.715[2]$ & $0.711[2]$ & $0.717[2]$ & $0.717[3]$ \\
\hline SVC [linear] & 0.710 & 0.609 & 0.505 & 0.600 & 0.640 \\
\hline SVC [poly] & 0.698 & 0.607 & 0.517 & 0.599 & 0.616 \\
\hline SVC [sigmoid] & 0.620 & 0.551 & 0.470 & 0.540 & 0.553 \\
\hline SVC [rbf] & 0.642 & 0.521 & 0.487 & 0.567 & 0.581 \\
\hline Decision Tree & 0.715 & 0.715 & 0.711 & 0.715 & 0.714 \\
\hline
\end{tabular}



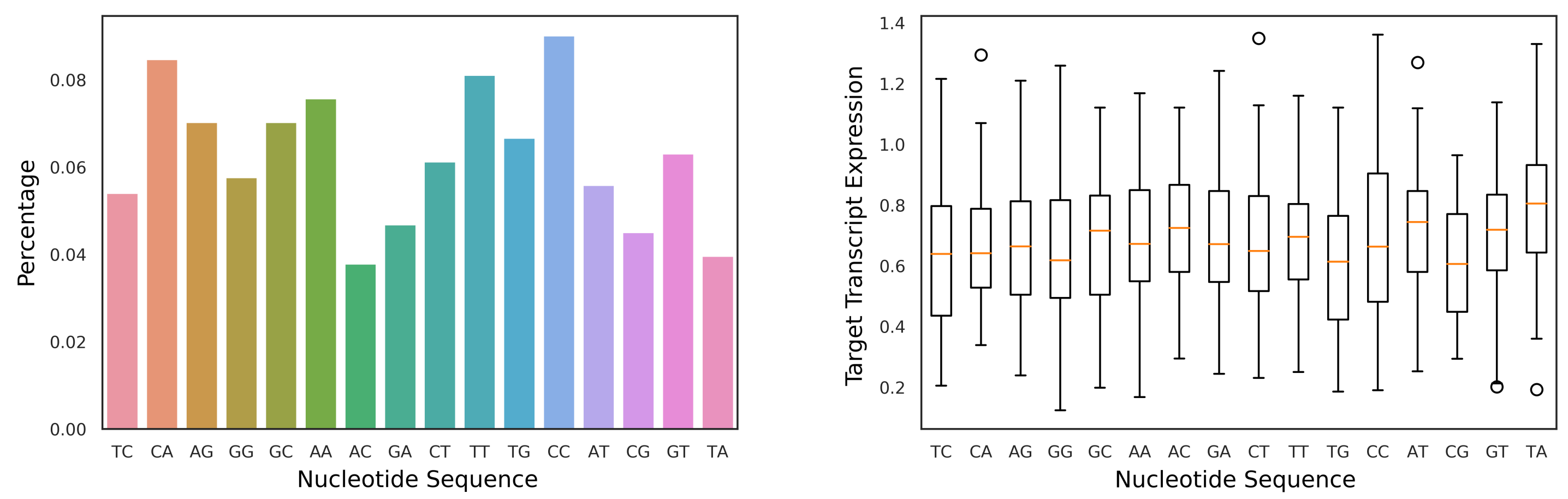

C

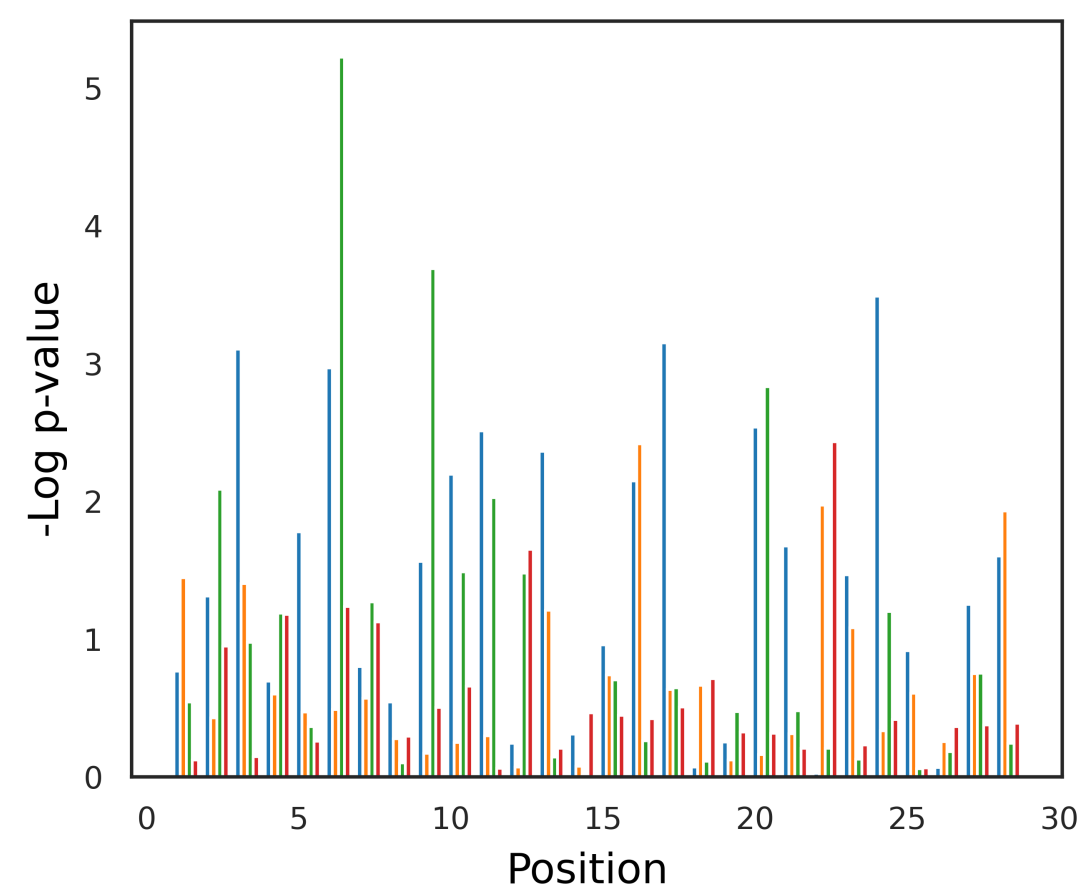

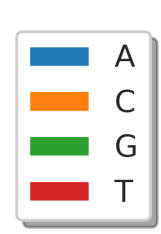

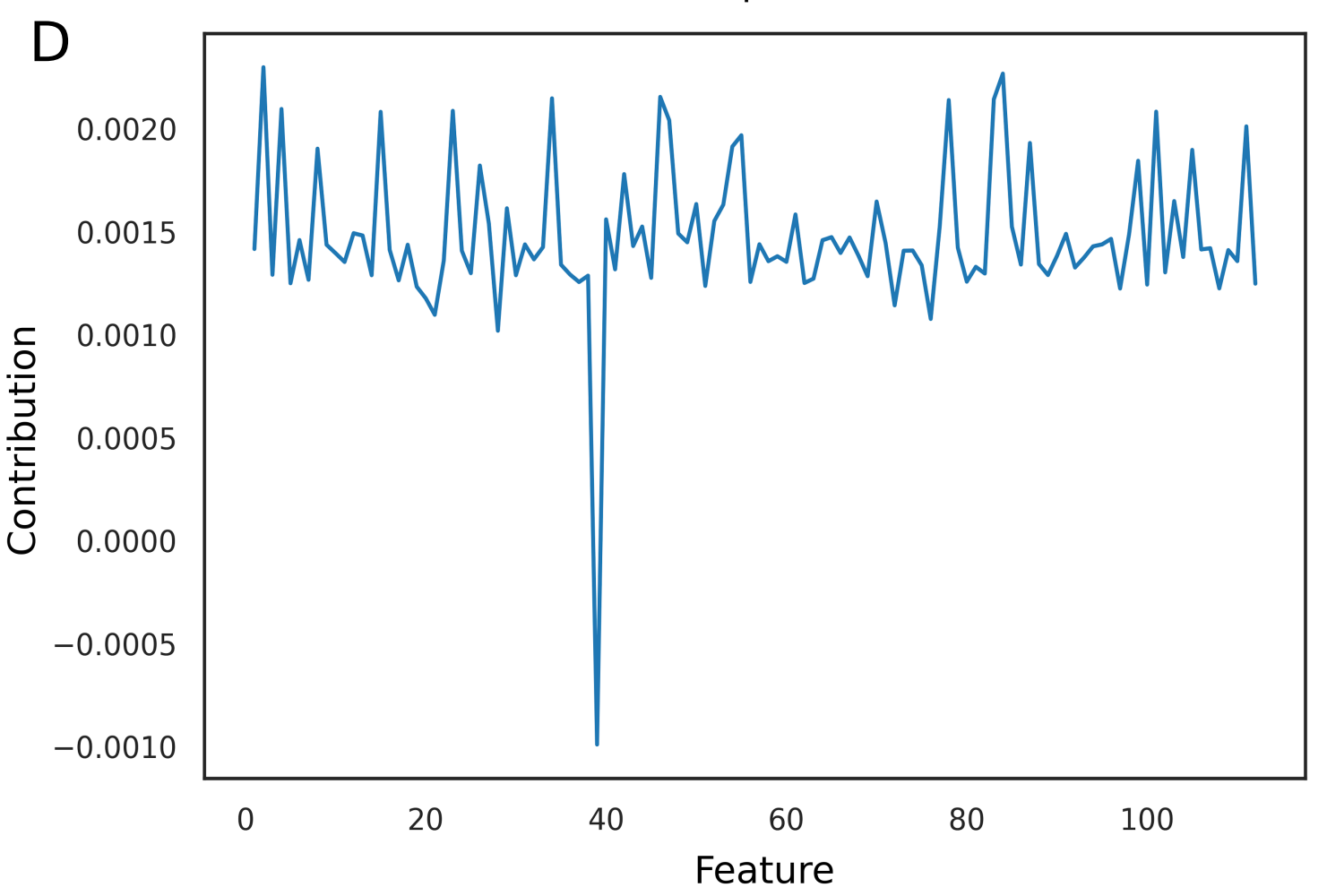




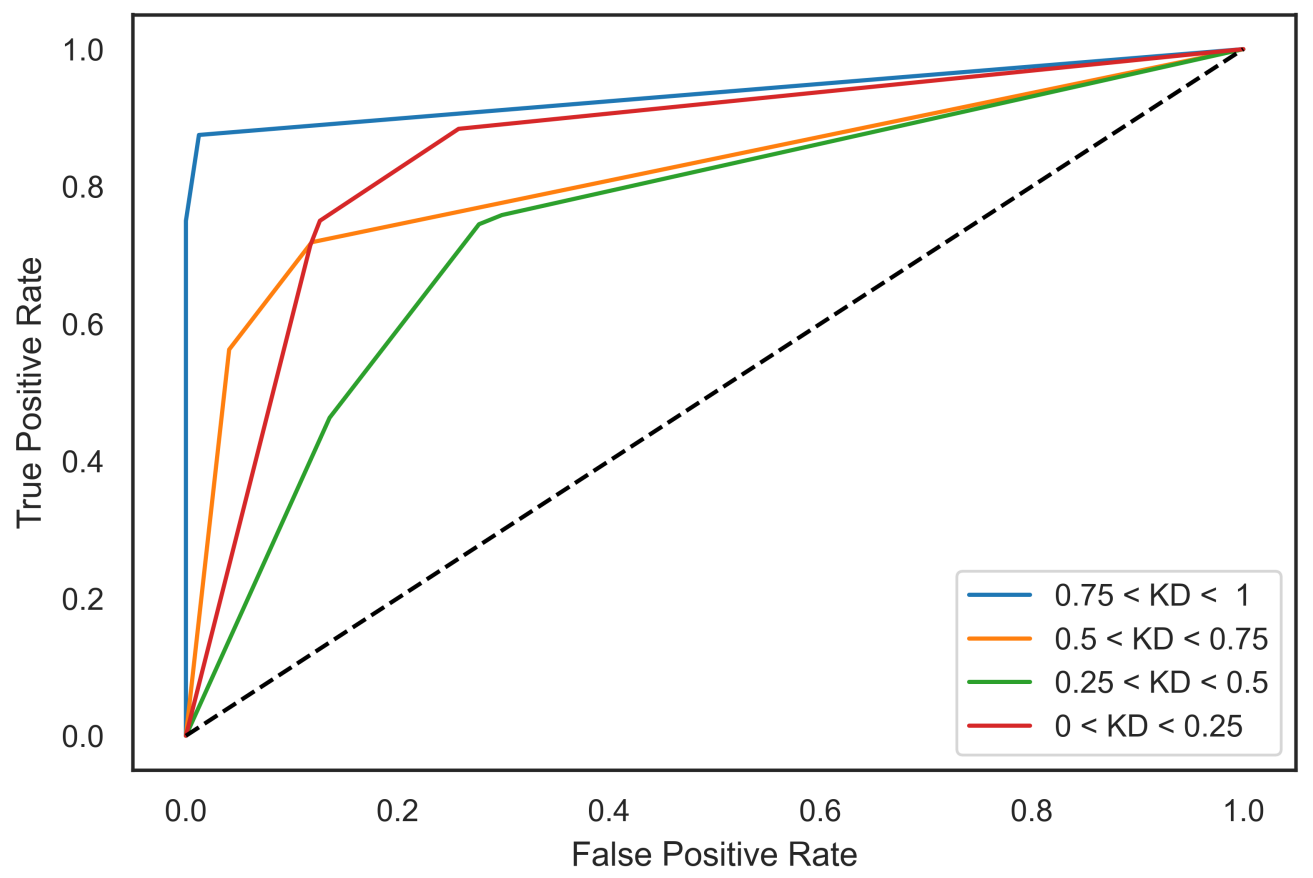




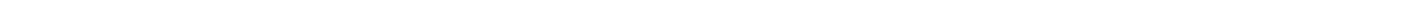


Protein Occupancy

Thance

Protein Occupancy

$\stackrel{\frac{\pi}{d}}{I} \quad$ Good Guides

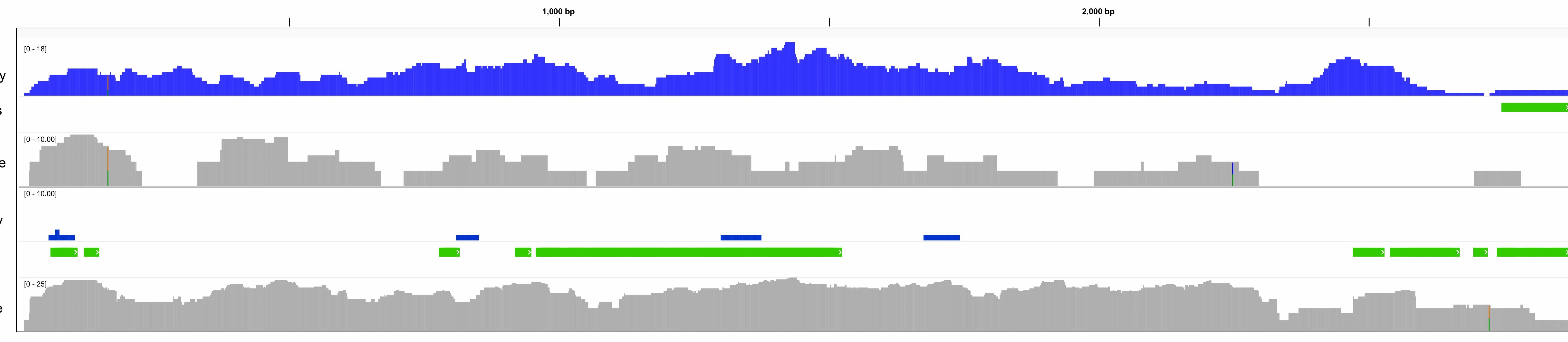

